\title{
(2) OPEN ACCESS \\ What does the ideal urgent and emergency care system look like? A qualitative study of service user perspectives
}

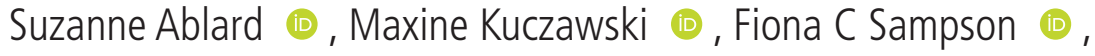 \\ Suzanne M Mason
}

Centre for Urgent and Emergency Care Research (CURE), SCHARR, University of Sheffield, Sheffield, UK

\section{Correspondence to} Mrs Maxine Kuczawski, Centre for Urgent and Emergency Care Research (CURE), ScHARR

University of Sheffield, Sheffield S1 4DA, UK:

m.kuczawski@sheffield.ac.uk

Received 11 July 2019 Revised 17 December 2019 Accepted 19 December 2019 Published Online First 9 January 2020

\section{Check for updates}

(c) Author(s) (or their employer(s)) 2020. Re-use permitted under CC BY-NC. No commercial re-use. See rights and permissions. Published by BMJ.

To cite: Ablard $\mathrm{S}$,

Kuczawski M, Sampson FC, et al. Emerg Med J

2020:37:200-205

\begin{abstract}
Background Policies aimed at diverting care from EDs to alternative services have not been successful in reducing $E D$ attendances and have contributed to confusion for service users when making care-seeking decisions. It is important that service users are at the heart of decision making to ensure new services meet the needs of those who will be accessing them. In this study, service users were encouraged to think freely about the desirable qualities of an ideal urgent and emergency care (UEC) system.
\end{abstract}

Methods From September to February 2019, an open inductive methodology was used to conduct focus groups with service users who had used UK UEC services within the previous year. Service users that had contact with NHS111, ambulance service, General Practice outof-hours, minor injuries unit, walk-in centre or ED were purposively sampled and stratified into the following groups: (1) 18-45 years; (2) $\geq 75$ years; (3) adults with young children; (4) adults with long-term conditions. Focus groups were structured around experiences of accessing UEC services and perspectives of an 'ideal' UEC system.

Results 30 service users took part in the study, across four focus groups. The ideal UEC system centred around three themes: a simplified UEC system (easier to understand and a single-point of access); more 'joinedup' UEC services and better communication between health staff and patients.

Conclusion Desirable qualities of an ideal UEC system from a service user perspective related to simplifying access for example, through a single point of access system where health professionals decide the appropriate service required and improving continuity of care through better integration of UEC services. Service users value reassurance and communication from health professionals about care pathways and care choices, and this helps service users feel more in control of their healthcare journey.

\section{INTRODUCTION}

Globally, demand for urgent and emergency care (UEC) services has increased significantly over the past two decades, partly due to an ageing population, but also due to service users attending services which are not the most appropriate for their health needs. ${ }^{12}$ Alternative UEC services which are designed to see and treat service users with urgent but non-life threatening health conditions have been set up as an alternative to the ED.

\section{Key messages}

What is already known on this subject

- Evidence has shown that policies aimed at shifting care away from EDs into alternative urgent care services have not achieved their intended effect of decreasing ED visits; as rather than helping people get to the most appropriate place for their health needs, they have resulted in further confusion.

- It is becoming more widely acknowledged that service users are well placed to comment on changes to health services as they have the advantage of being able to take a whole systems perspective rather than focussing on individual services within the system; therefore being able to identify which elements of the system could benefit most from improvement to enable more streamlined access.

\section{What this study adds}

- In this qualitative study of recent users of unscheduled care, there was confusion around the appropriate usage of services within the UEC system, with service users stating preference for a single point of access (which includes a number of co-located urgent and emergency care services) within which health professionals decide which service is most appropriate for the service user based on their presenting complaint.

- Service users agreed that health services need to work more closely together to provide an efficient and joined up service; which may be supported by creating nationwide linked medical records.

- Service users want reassurance early in their care pathway, and were prepared to accept long waits as long as they were given information about what was happening to them and why.

In the UK, these include: co-locating primary care services with EDs, ${ }^{3}$ walk-in centres, ${ }^{4}$ and telephone triage systems (eg, NHS 111). ${ }^{5}$ However, evidence shows these services have not achieved their intended effect of decreasing ED visits ${ }^{6}$; but have led to further confusion about the most appropriate services for particular health needs. ${ }^{7}$ 
To understand how services will be accepted and used, the NHS long-term plan emphasises the importance of putting service users at the heart of decision making. ${ }^{8}$ Service users are uniquely placed because they can comment on their journey through the whole UEC system, rather than focussing on individual services; therefore being able to identify which elements could benefit from improvement to enable more streamlined access to the help they need. ${ }^{9}$ While a number of studies have explored how service users negotiate their way through the UEC system, ${ }^{10-13}$ and described the factors appearing to influence patient satisfaction ${ }^{914-16}$; there is a paucity of studies directly asking service users what they would like from an ideal UEC system. Discussions about what service users want from the UEC system would help ensure new services are designed to meet the needs of service users who will be accessing them.

We undertook a study whereby participants were encouraged to think freely about the essential and desirable qualities of an ideal UEC system. Specifically, we aimed to explore service users' positive and negative experiences of accessing the UEC system and the key characteristics of an ideal UEC system.

\section{METHODS}

\section{Design and setting}

We used an inductive approach and conducted focus groups with a purposive sample of service users who had accessed services within the UEC system. This methodology was used as it offers a degree of flexibility, whereby the researcher takes on a less directive and dominating role, enabling participants to comment on areas they believe are most important to them within a focussed discussion.

The study was undertaken in a large urban English city (Sheffield) between July 2018 and February 2019. The UEC system in Sheffield includes an adult-only (16 years and older) Type 1 ED which has an out-of-hours Primary Care Centre (accessible via NHS 111) located on the same site. There is a stand-alone Children's Hospital with an ED (0-15 years) and two separately located UEC centres in the City Centre a minor injuries unit and a primary care-led walk-in centre. Sheffield is served primarily by one ambulance service. Service users can also access a national healthcare telephone advice service (NHS 111); a 24 hours, 7 days a week service intended for urgent but not life-threatening' health issues.

\section{Sampling}

Service users who fell into one of four cohorts (18-45 years; 75 years and over; adults with young children aged 5 years or under; and adults with long-term conditions) and had accessed the UEC system in Sheffield within 12 months prior to recruitment were eligible to participate. These cohorts were selected as they have been reported as accessing UEC services differently with higher rates of unnecessary attendance to UEC services. O'Keeffe et al reported significantly higher odds of non-urgent attendance to the ED for both younger patients (aged 16-44) and over 65 compared with those aged 45-64 years (OR 1.42 and 3.81 respectively). ${ }^{17}$ Increased non-urgent attendance to UEC services has also been reported in young children ${ }^{18} 19$ and those with long term conditions such as asthma, diabetes and mental illness. ${ }^{20}$

\section{Recruitment}

A range of recruitment techniques were employed to identify participants such as face to face, online advertising (Peopleinresearch.org; Gumtree; Facebook) email distribution lists and posters displayed in public areas. These techniques were used in the City Centre, public spaces (supermarkets, libraries, etc) and within the UEC services of interest. Members of the study team also approached community support groups where our target cohorts were known to attend (parent and toddler, coffee mornings) and information about the study was either distributed or a member of the study team visited to discuss the study.

Service users expressing an interest in participating were sent a study pack including a cover letter, information sheet, eligibility questionnaire and reply slip. The eligibility questionnaire included contact details, age group, long-term conditions, UEC services accessed in the previous 12 months and any children living with the responder. On completion of the reply slip and eligibility questionnaire a member of the study team contacted the service user to confirm the details of the focus group.

\section{Data collection}

We used an open, inductive approach, with just two key questions to guide discussions during focus groups: (1) would anyone like to share their positive and negative experiences of accessing services within the UEC system? (2) In an 'ideal world' (ie, there are no funding constraints), what would your ideal UEC system look like? By asking service users to reflect on their previous encounters with the UEC system, it ensured the ideal UEC system generated was grounded in the service user's personal experiences and focussed on what was important to them rather than being influenced by the focus group facilitators.

Service users provided written informed consent at the beginning of the focus group. Focus groups were facilitated by two of the authors (SA and MK), were audio-recorded and took place in a private room at the University of Sheffield. The duration of the focus groups was 3 hours with a 20 min break; participants were asked the two key questions with time given to fully explore participants' responses and discuss among the group. During the focus groups, service users were provided with a buffet lunch or dinner and on completion they received a $£ 30$ shopping voucher to compensate them for their time.

\section{Patient and public involvement (PPI)}

The Sheffield Emergency Care Forum (SECF) PPI group were involved from the early stages of the study; the study team worked with the group who advised on the key questions, structure of the focus groups (including length and incentive offered), development of the study materials (poster, leaflet) and participant recruitment. Members of the SECF assisted with the displaying of study material and informing potential participants about the study. During the final report writing stage, the SECF reviewed the study findings and provided advice regarding the writing style and plain English language.

\section{Analysis}

Focus group discussions were transcribed verbatim; as we were trying to understand service user perspectives of what was important to them, without preconceived ideas, we undertook an inductive approach to analysis, ensuring that themes were identified from the data themselves, rather than fitting into a pre-existing coding framework. The research was influenced by the paradigm of pragmatism, which focusses on 'what works' and values both subjective and objective knowledge. 2122

Reflexivity was performed to recognise and reflect the role of the researcher within the undertaking of the focus groups, and analysis of the data. Three of the research team (SA, MK and FCS) are social scientists with no clinical experience of working 


\begin{tabular}{|c|c|c|c|c|}
\hline \multirow[b]{2}{*}{ Cohort } & \multicolumn{4}{|l|}{ Focus group } \\
\hline & 1 & 2 & 3 & 4 \\
\hline $18-45$ years & 5 & 2 & 1 & 2 \\
\hline 75 years + & 0 & 1 & 0 & 0 \\
\hline $\begin{array}{l}\text { Parent with } \\
\text { young child }\end{array}$ & 2 & 1 & $2^{*}$ & 1 \\
\hline $\begin{array}{l}\text { Adults with } \\
\text { long-term } \\
\text { condition }\end{array}$ & $4 \dagger$ & $4 \ddagger$ & $3 \S$ & $4 \uparrow$ \\
\hline $\begin{array}{l}\text { Services } \\
\text { accessed } \\
\text { within the last } \\
12 \text { months }\end{array}$ & $\begin{array}{l}\text { Minor injuries } \\
\text { unit; GP-led } \\
\text { walk-in centre; } \\
\text { ED; ambulance } \\
\text { service; NHS } \\
111\end{array}$ & $\begin{array}{l}\text { ED; GP-led } \\
\text { walk-in } \\
\text { centre }\end{array}$ & $\begin{array}{l}\text { Minor-injuries } \\
\text { unit; ED; NHS } 111\end{array}$ & $\begin{array}{l}\text { ED; NHS 111; } \\
\text { ambulance } \\
\text { service; GP-led } \\
\text { walk-in centre; } \\
\text { Minor injuries } \\
\text { unit }\end{array}$ \\
\hline $\begin{array}{l}\text { Total service } \\
\text { users }\end{array}$ & 11 & 6 & 6 & 7 \\
\hline
\end{tabular}

*Includes two service users aged $18-45$ years.

tIncludes one service user who was the parent of a young child.

‡Includes one service user aged $18-45$ years.

§Includes one service user aged $18-45$ years and two service users aged 75 years or older.

IIncludes one service user aged $18-45$ years and one service user aged 75 years or older.

within the UEC system, but with previous experience of research within the area of UEC systems. SMM is an emergency consultant with significant research experience within the area of UEC systems.

A coding framework was developed after discussion with the research team, and initial coding was undertaken by one researcher (SA). Developing themes and emerging interpretations were discussed among the research team. NVivo V.12.0 (QSR International (UK) Limited, Daresbury, UK), ${ }^{23}$ was used to help structure the analysis. Data saturation was reached as the core themes generated from the data were consistent across all focus groups.

\section{RESULTS}

\section{Sample characteristics}

Thirty service users took part across four focus groups. Focus group sizes ranged from six to 11, taking place between September 2018 and February 2019 and included mixed cohorts partly due to logistical problems with arranging separate groups for different users but also because some service users stratified into more than one cohort (ie, aged 75 years or older and had a long-term condition) (table 1 ).

\section{Overview of themes}

The ideal UEC system was centred on three themes (table 2).

1. A simplified UEC system which is easier to understand and a single-point of access.

2. A more ‘joined-up' UEC system.

3. Better communication from health staff to patients when accessing UEC services.

\section{A simplified UEC system which is easier to understand and a single- point of access}

Dissatisfaction with health services was directed towards the system (eg, location of services) rather than the clinical care or clinicians who were treating them.

A story you hear quite a lot when you speak to people, it's the system and the processes that are, seem stressful but when you actually see the professional they are outstanding! (25-34 years; parent with young child)

While many service users (particularly those with long-term conditions) said they would prefer to see their own GP, as they receive the continuity of care not afforded to them in other parts of the system, difficulties getting an appointment within a timely manner meant they had to seek care elsewhere.

2 weeks it takes me to get an appointment, 2 weeks it would be for one! It's a joke, you're having a laugh. (55-64 years; long-term condition)

Service users expressed frustration at having to negotiate access to GP's via a receptionist who has no medical training and who was perceived as being unable to make a judgement about the seriousness of their health condition, thereby preventing easy access to the help they need.

One of the things I'd like to see, is your receptionist having a bit of medical knowledge. I'm not saying being as trained up as a doctor or a nurse, but, cos if you want triaging at our place you've got to actually tell the receptionist what's wrong with you and what is the point of telling them when they ain't got a clue what you're talking about. They'll just put it on computer. (45-54 years; longterm condition)

Service users said they want GP practices to extend opening times to accommodate patient demand.

I don't know why GPs shut on Saturday and Sundays because people don't stop being ill at the weekend. (45-54 years; long-term condition)

Table 2 Overview of the themes and subthemes from the focus groups

\begin{tabular}{l} 
Theme \\
\hline (1) A simplified UEC \\
system which is easier to \\
understand and a single- \\
point of access \\
(2) A more 'joined-up' UEC \\
system
\end{tabular}
Subthemes

Service users want clear and up to date information about the services available to them in their local area Service users want equal access to services within the UEC system regardless of where they live

Service users want the responsibility of where to be seen taken away from the service user and put into the hands of health professionals who would then triage them to the most appropriate service based in one place.

Service users indicated they want linked medical records that all services within the UEC system can access so that they receive continuity of care regardless of what point they access the UEC system and do not have to repeat medical information.

Service users expressed confidence in the NHS' ability to protect their data but the linkage of patient records within individual services was seen as a greater priority which overrode their concerns around data security

(3) Better communication from health staff when accessing UEC services

Service users want to be an active participant in their healthcare journey with more information about what is happening to them and why Communication about waiting times was more important to services users than the wait times themselves. 
Service users were aware some health conditions did not warrant the services of an ED, but equally they could not wait until a GP appointment became available. While they did not want to burden overstretched EDs; due to a lack of information or knowledge about alternative services they did not feel confident they were able to make the correct choice.

I think there's too much. It's great that there are different services for different levels of care but that's not helpful if people don't know when to use what. (25-34 years; parent with young child)

Some of the difficulties there are that people are not always the best witnesses of their own distress or illness. They're not very good at reporting so....or find it very difficult to articulate. (45-54 years; long term condition)

Service users described unequal access to services within the UEC system. For example, service users living a greater distance away from the ED experienced considerable barriers to access, particularly if they did not have personal transport (eg, a car); whereas service users liked the central location of the walk-in centre because it is accessible to all and has good public transport links.

I live way down in the South of the city and if I had an aortic aneurysm I would never have got to [name of hospital] alive ok ... That's one thing that I really do worry about. It's a long journey to get there, very traffic dependent whether you've got the two's and blues on or not, it takes time. (25-34 years)

In an ideal world, service users would like multiple UEC hubs spread out across the city so no one has to travel too far to get access to the help they need. Each of these hubs would have co-located services (including minor injuries, GP out-of-hours, ED) based on the same site. When someone enters the hub they would be streamed to the most appropriate service based on their health complaint by a health professional, as participants appeared to feel insecure about making their own decisions on where to go. Since the services would be based on one site it would be easy to switch between services if they were initially streamed to the wrong place.

Maybe we should have one in the East and one in the West or there should just be more than one....everyone should be able to get to this magic centre, whatever it's gonna be, within a certain time frame. (25-34 years; long term condition)

I think that the decision of where to go to shouldn't be made by you. It should be made by somebody that you see in the first place. The first point of call is some kind of reception, huge reception with multiple desks. (25-34 years; parent with young child)

I think everything in the same building is good cos then if you get referred you just walk like down the corridor or something. (25-34 years; long term condition)

Yeah, in an urgent care situation, the easiest route is the best route. (25-34 years; parent with young child)

Service users were keen to have a community transport system accessible to people who are unable to get to the hospital independently but who are not serious enough to warrant an ambulance.

It's that thing of your not independently having to travel on a bus, you can just ring up. As you say you might ring up through 111 and they go yes, ok, someone will be with you in $5 \mathrm{~min}$, you know $10 \mathrm{~min}, 15 \mathrm{~min}$. It's not urgent but yes being accompanied to make sure you're all right and your safe. It's not life threatening, life threatening. So it's a situation where you wouldn't ring 999 but equally you need immediate attention. (25-34 years; long term condition)

\section{A more 'joined-up' UEC system}

Frustrations were raised about the UEC system not having access to GP records, resulting in service users repeating key information (eg, medications, allergies) at every encounter with a clinician. Since service users are so connected in other areas of their lives, there was an assumption that health services should be connected in this way too and could not comprehend why this was not the case.

With tablets and things like that. Cos you call your doctor and they want to know all this and the other and then paramedics come out and they want to know all that. Then you get to A\&E and they want to know all that. You get to the cubicle and they want to know all that. (65-74 years; long term condition)

Service users want a nationwide computer system which links together health services, so that whatever health service they access, health professionals will have a copy of their medical history to ensure continuity of care.

I think it would be nice if there was one centralised system for everyone for the whole of NHS, GP, everyone. Just so that when you go to A\&E they can access everything. (25-34 years; long term condition)

Service users were asked whether they had concerns around data security but none were raised. While service users expressed confidence in the NHS' ability to protect their data; the linkage of services was seen as a greater priority which overrode concerns around data security. However, there were limits to the sharing of health information, with some stating they would not want this shared with external organisations.

I think we should have access to it, but then I'm also thinking, data breaches, the amount of, security attacks, cyber attacks, are you then going to, with your lack of funding for the NHS, if everything then goes digital, how many people are then going to sue the NHS because of data breach? Because someone left a USB on a bus? How secure are the connections? Personally I don't mind people having that data, if I'm in an emergency... (25-34 years; young adult)

I have concerns about information sharing. I don't want my medical data to be shared with the likes of insurance companies. It's medical! (35-44 years)

Some service users lacked confidence in the NHS' ability to create nationwide linked medical records. To overcome this, service users discussed the option of being in control of their own medical records (perhaps in the form of a USB or electronic bracelet), so when they access services they can hand over their health information to help facilitate continuity of care.

Well we have got mobile phones with, you know, like personal details, we have access to banking and things like that on our phones, almost like we should have, hold our own records. (25-34 years; parent with young child).

\section{Better communication from health staff to patients when accessing} UEC services

Service users shared stories about waiting for treatment for long periods of time with no information about what is happening and why, with feelings of anxiety exacerbated when services users were unaware of the standard journey through the ED. Assertiveness was described as a way to get more information but not all service users felt confident doing this for fear of being perceived as a nuisance. Once seen by a health professional, the clinical care received was often described as excellent; it was the lack of information between assessments that caused greatest concern. 
I didn't know what the next step was. Like I didn't know that I was gonna be going into a little consultation room and having a triage, kind of, well I think that was where they were deciding who I was gonna see and whether it was needed or not, or whether they could send me home. But I just didn't know, and then I was told after doing the triage, it was like, well a doctor needs to see you. I was like, you're not a doctor? What are you? Who have I just spoken to? (25-34 years; parent with young child)

Service users justified long waits due to health professionals being under considerable pressure to see an increasing number of attendances; with an acceptance that other service users may be seen quicker because they are considered more urgent. Being kept updated about waiting times was considered more important than actual wait times.

Waiting times is long at hospital but they've got a hard job, a really hard job. They do well, so I commend them! (55-64 years; longterm condition)

If somebody came every half an hour and said sorry you've been waiting, you're third in the queue or whatever. Just a few updates. (65-74 years; long-term condition)

One solution proposed by service users was to have 'floor walkers' in waiting rooms who could set expectations about what is happening, check health complaints do not worsen and ensure the environment remains a safe space to wait.

I think having someone or a couple of people who are walking around. I dare say, patrolling the area that people are waiting, checking in with people. Again going back to managing expectations, keeping the environment calm, and helping people to kind of get through their time waiting. And using that to spot where there might be trouble brewing or where people might be getting agitated and being able to step in and deal with them before they get escalated. (35-44 years)

Other ideas included technological solutions to update service users where they are in the system and what the approximate wait times are; something visual to look at so they know they have not been forgotten. Service users discussed how big corporations are already using similar systems and queried whether they could be incorporated into health services.

What about an app that tells you the waiting times? I mean they do it at Alton Towers because I was there a few weeks ago. You can look at each one of the rides and think oh it's an hour and a half right, we won't do that one. (35-44 years; parent with young child)

However, technological solutions were not favoured by all

No. Terrifying! Put my hand up. Help! (over 75 years; long-term condition)

\section{DISCUSSION}

Ideas centred around three themes: (1) a simplified UEC system which is easier to understand and a single-point of access; (2) a more 'joined-up' UEC system; (3) better communication from health staff to patients when accessing UEC services. These ideas were directly influenced by service users past experiences of accessing the UEC system, whereby they attempted to identify solutions to problems encountered.

There are similarities to our research findings in literature relating to service user experience and satisfaction with services. ${ }^{914-16} \mathrm{~A}$ literature review found service users longed for more information from health professionals and became anxious when they were left alone for long periods of time; information about waiting times was more influential on the service user experience than the waiting time itself; and service users understood that people who were more critically ill than themselves would be seen as a priority. ${ }^{16}$ As in our study, service users are surprisingly accepting of long waits as they understand the health system is overstretched and prioritisation needs to take place, but what is important is that they are kept informed about what is happening to them and why. Service users were also keen to receive reassurance early in their clinical care pathway, especially if their condition is not considered (by clinicians) to be immediately 'life threatening' and that they are safe to be in the waiting room.

Frustrations experienced by service users in having to repeat their past medical history during numerous encounters with health professionals across the UEC system is not unique to our study. ${ }^{24}$ Solutions to this problem are being addressed in a number of government reports with plans to create personalised comprehensive care records for individuals that contain sufficient information to inform health professionals in their decision making about service users wherever they are seen. ${ }^{8}{ }^{24}$ Service users in our study expressed overwhelming trust in the NHS' ability to protect their health data. However, this trust may be overstated when taking into consideration their strong desire for a more joined up UEC system and the fact that conversations were positioned in the context of an 'ideal world' where service users may assume breaches of data security are not a threat. Outside the focus group setting policy makers may encounter resistance to the sharing of health information unless concerns around data security are clearly addressed, especially when it comes to sharing personal health data beyond NHS-led organisations.

Too often the impetus for creating new services has been to re-direct patients into what is deemed a more appropriate health service based on their presenting complaint. Evidence has shown policies aimed at shifting care away from EDs into alternative urgent care services have not achieved their intended effect of decreasing ED visits $^{6}$; as rather than helping people get to the most appropriate place for their health needs, these alternative services have resulted in further confusion. ${ }^{7}$ As shown in our study, service users do not want to deliberately burden the overstretched UEC system, but due to the multitude of obstacles encountered; it is perhaps no surprise they inadvertently end up using services in a manner labelled 'inappropriate'. Solutions designed by service users in our study involved taking the responsibility of where to be seen away from the service user, and putting it into the hands of the clinicians who will be treating them; so when someone is sick or injured all they are confronted with is a single point of access, within which they are streamed to the most appropriate place based on their health need.

Our study highlighted the importance of involving service users in the design of the UEC system. Service users are well placed to comment on the UEC system as they are able to take a whole systems approach, rather than focussing on individual services. Service users find it particularly difficult talking about services in isolation of each other and prefer to describe their experiences as a journey involving multiple decisions, encounters with health professionals and obstacles to overcome. By taking this approach, it is apparent that what happens in one section of the UEC system can have significant consequences later on. Policy makers and researchers should increase the involvement of service users in discussions around the reconfiguration of the UEC system, to ensure any changes made will be directly beneficial to those accessing it.

\section{Limitations}

Because the structure of the focus groups was designed to encourage participants to reflect on their past experiences, this may have encouraged users to focus overly on the UEC system as it exists now, constraining any 'blue sky' thinking. 
Every attempt was made to optimise recruitment for the study by employing a multitude of techniques. However difficulties were experienced; the study team was unable to recruit from the waiting room of The Children's Hospital due to delays in the approvals process and the final participant sample contained an under-representation by parents with young children and people aged over 75 years. The use of targeted recruitment to specific groups may also have led to potential selection bias. Reasons for non-recruitment may include lack of childcare or accessibility issues (eg, some over 75's may be housebound), which we were unable to overcome. When designing alternatives to the current UEC system it is important to include representation from all groups within society, including those that are traditionally hard to reach (eg, the homeless). This should be explored further in future research.

Finally, the research was undertaken on a limited number of participants in a large urban English city and therefore previous experience of the UEC system that participants referenced was based largely on the context of the NHS. The study findings thus may not reflect the thoughts of service users based elsewhere in the UK, and may limit transferability of findings to settings outside the UK.

\section{CONCLUSION}

Participants within this study identified three main elements to an ideal UEC system; a simplified UEC system with a single-point of access, a more 'joined-up' UEC system and better communication from health staff when accessing UEC services. Service users found that too many individual UEC services, and a lack of communication between them to be confusing and frustrating. Service users expressed a desire for individual UEC services to work closer together to provide an efficient and more 'joined-up' service, with a single point of access where health professionals decide the appropriate service required. Improved communication from health professionals to service users about what is happening and why would help service users feel more in control of their healthcare journey and provide reassurance. Future research should focus on understanding what communication service users prefer, when and from whom to help support them on the care pathway.

Twitter Fiona C Sampson @fcsampson and Suzanne M Mason @ProfSueMason

Acknowledgements The authors would like to thank the Sheffield Emergency Care Forum (SECF) PPI group (https://secf.org.uk/) for their helpful feedback on the recruitment materials (cover letter, information sheet, leaflet and poster) developed for this project to ensure they were suitable for a lay audience and for helping the research team in distributing leaflets and posters advertising the study. We also thank our participants for giving up their valuable free time to share with us their experiences of accessing the UEC system, and for helping us to understand what it is they would like from an ideal UEC system.

Contributors SA, MK and SMM contributed to the design of the work. SA and MK facilitated the focus groups and undertook the analysis and interpretation of data. All authors (SA, MK, FCS, SMM) contributed to drafting the work or revising it critically for important intellectual content.

Funding The research was funded by the National Institute for Health Research (NIHR) Collaboration and Leadership in Applied Health Research and Care, Yorkshire and Humber (CLAHRC YH): Avoiding Attendances and Admissions Theme (AAA) and Connected Health Cities which is a Northern Health Science Alliance Programme funded by the Department of Health and Social Care.

Competing interests None declared.

Patient consent for publication Not required.

Ethics approval A UK National Research Ethics Committee granted ethical approval for the conduct of the research (18/NS/0076).

Provenance and peer review Not commissioned; externally peer reviewed.

Data availability statement No data are available. It is not possible to fully anonymise the qualitative focus group transcripts and therefore no additional data is available.
Open access This is an open access article distributed in accordance with the Creative Commons Attribution Non Commercial (CC BY-NC 4.0) license, which permits others to distribute, remix, adapt, build upon this work non-commercially, and license their derivative works on different terms, provided the original work is properly cited, appropriate credit is given, any changes made indicated, and the use is non-commercial. See: http://creativecommons.org/licenses/by-nc/4.0/.

\section{ORCID iDs}

Suzanne Ablard http://orcid.org/0000-0001-5340-9510

Maxine Kuczawski http://orcid.org/0000-0002-0774-8113

Fiona C Sampson http://orcid.org/0000-0003-2321-0302

Suzanne M Mason http://orcid.org/0000-0002-1701-0577

\section{REFERENCES}

1 Barker C. Accident and emergency statistics: demand, performance and pressure. house of commons library. Briefing paper 6964, 2017. Available: https:// researchbriefings.parliament.uk/ResearchBriefing/Summary/SN06964 [Accessed 11 Jul 2019].

2 Durand A-C, Gentile S, Devictor B, et al. ED patients: how nonurgent are they? Systematic review of the emergency medicine literature. Am J Emerg Med 2011;29:333-45.

3 Ramlakhan S, Mason S, O'Keeffe C, et al. Primary care services located with EDS: a review of effectiveness. Emerg Med J 2016:33:495-503.

4 Arain M, Nicholl J, Campbell M. Patients' experience and satisfaction with GP led walk-in centres in the UK; a cross sectional study. BMC Health Serv Res 2013;13:142.

5 Turner J, O'Cathain A, Knowles E, et al. Impact of the urgent care telephone service NHS 111 pilot sites: a controlled before and after study. BMJ Open 2013;3:e003451.

6 Tan S, Mays N. Impact of initiatives to improve access to, and choice of, primary and urgent care in England: a systematic review. Health Policy 2014;118:304-15.

7 Pope C, McKenna G, Turnbull J, et al. Navigating and making sense of urgent and emergency care processes and provision. Health Expectations 2019;22:435-43.

8 NHS. The NHS long term plan, 2019. Available: https://www.longtermplan.nhs.uk/wpcontent/uploads/2019/01/nhs-long-term-plan.pdf [Accessed 26 Apr 2019].

9 Patient Opinion. Patient experiences of urgent and emergency care in Yorkshire and the Humber: an analysis of stories from patient opinion, 2015. Available: http:// s3-eu-west-1.amazonaws.com/yhahsn.org.uk/wp-content/uploads/2015/09/PatientOpinion-emergency-care-report-June-2015.pdf [Accessed 22 Mar 2018].

10 O'Cathain A, Coleman P, Nicholl J. Characteristics of the emergency and urgent care system important to patients: a qualitative study. J Health Serv Res Policy 2008;13:19-25.

11 Coleman P, Irons R, Nicholl J. Will alternative immediate care services reduce demands for non-urgent treatment at accident and emergency? Emerg Med J 2001;18:482-7.

12 Penson R, Coleman P, Mason S, et al. Why do patients with minor or moderate conditions that could be managed in other settings attend the emergency department? Emerg Med J 2012;29:487-91.

13 Knowles $E$, O'Cathain A, Nicholl J. Patients' experiences and views of an emergency and urgent care system. Health Expectation 2012;15:78-86.

14 Nairn S, Whotton E, Marshal C, et al. The patient experience in emergency departments: a review of the literature. Accid Emerg Nurs 2004;12:159-65.

15 Taylor C, Benger JR. Patient satisfaction in emergency medicine. Emerg Med J 2004;21:528-32.

16 Gordon J, Sheppard LA, Anaf S. The patient experience in the emergency department: a systematic synthesis of qualitative research. Int Emerg Nurs 2010;18:80-8.

17 O'Keeffe C, Mason S, Jacques R, et al. Characterising non-urgent users of the emergency department (ED): a retrospective analysis of routine ED data. PLoS One 2018;13:e0192855

18 McHale P, Wood S, Hughes K, et al. Who uses emergency departments inappropriately and when - a national cross-sectional study using a monitoring data system. BMC Med 2013:11:258.

19 Viner RM, Blackburn F, White F, et al. The impact of out-of-hospital models of care on paediatric emergency department presentations. Arch Dis Child 2018;103:128-36 https://doi.org/

20 Deeny S, Thorlby R, Steventon A. Briefing: reducing emergency admissions: unlocking the potential of people to better manage their long-term conditions. London, UK: The Health Foundation, 2018. https://www.health.org.uk/publications/reducingemergency-admissions-unlocking-the-potential-of-people-to-better-manage-theirlong-term-conditions

21 Pansiri J. Pragmatism: a methodological approach to researching strategic alliances in tourism. Tourism and Hospitality Planning \& Development 2005;2:191-206.

22 Cresswell JC, Plano Clark VL. Designing and conducting mixed methods research. 2nd edn. Thousand Oaks California: Sage publications, 2011: 43.

23 QSR International Pty Ltd.. NVivo qualitative data analysis software (version 12), 2018.

24 NHS England. Local health and care records exemplar, 2018. Available: https:// www.england.nhs.uk/wp-content/uploads/2018/05/local-health-and-care-recordexemplars-summary.pdf [Accessed 4 Jun 2019]. 